\title{
Analysis of the stress-inducible transcription factor SsNAC23 in sugarcane plants
}

\author{
Renata Fava Ditt ${ }^{1}$, Agustina Gentile ${ }^{1}$, Rafael Garcia Tavares ${ }^{1}$, Sandra Rodriguez Camargo ${ }^{1}$, \\ Jorge Hernandez Fernandez ${ }^{2}$, Marcio Jose da Silva ${ }^{3}$, Marcelo Menossi ${ }^{1 *}$ \\ ${ }^{1}$ UNICAMP/Inst. de Biologia - Depto. de Genética, Evolução e Bioagentes - Lab. de Genoma Funcional, C.P. \\ 6109 - 13083-875 - Campinas, SP - Brasil. \\ ${ }^{2} U E N F / C B B / L Q F P P$ - 28013-602 - Campo dos Goytacazes, RJ - Brasil. \\ ${ }^{3}$ UNICAMP/Inst. de Biologia/Centro de Biologia Molecular e Engenharia Genética - Depto. de Genética, \\ Evolução e Bioagentes, C.P. 6109 - 13083-875 - Campinas, SP - Brasil. \\ *Corresponding author<menossi@lgf.ib.unicamp.br> \\ Edited by: Leonardo Oliveira Medici
}

\begin{abstract}
Stresses such as cold and drought can impair plant yield and induce a highly complex array of responses. Sugarcane (Saccharum spp.) is cultivated in tropical and subtropical areas and is considered a coldsensitive plant. We previously showed that cold stress induces the expression of several genes in in vitro sugarcane plantlets. Here we characterize one of those genes, $S s N A C 23$, a member of the NAC family of plant-specific transcription factors, which are induced by low temperature and other stresses in several plant species. The expression of $S s N A C 23$ was induced in sugarcane plants exposed to low temperatures $\left(4^{\circ} \mathrm{C}\right)$. With the aim of further understanding the regulatory network in response to stress, we used the yeast two-hybrid system to identify sugarcane proteins that interact with SsNAC23. Using SsNAC23 as bait, we screened a cDNA expression library of sugarcane plants submitted to $4^{\circ} \mathrm{C}$ for $48 \mathrm{~h}$. Several interacting partners were identified, including stress-related proteins, increasing our knowledge on how sugarcane plants respond to cold stress. One of these interacting partners, a thioredoxin h1, offers insights into the regulation of SsNAC23 activity.
\end{abstract}

Keywords: SsNAC, in situ hybridization, cold stress, yeast two hybrid

\section{Introduction}

Sugarcane (Saccharum spp.) is an important tropical grass crop that suffers from abiotic stresses caused by low temperatures and drought (Robertson et al., 1999; Tai and Lentini, 2002; Silva et al., 2008). These stresses can reduce sugarcane photosynthetic rates considerably, and a decrease by more than $50 \%$ was observed after exposure to temperatures of $10^{\circ} \mathrm{C}$ for 3 days (Du et al., 1999). This can lead to alterations in sugar quality and reduction in biomass.

Plants respond to cold stress with physiological changes and alterations of their gene expression, which can lead to higher levels of tolerance and cold acclimation (Guy et al., 1985; Tomashow, 1998; Allen and Ort, 2001). Genes regulated by cold are also commonly regulated by drought and salinity stresses (Seki et al., 2003; Shinozaki et al., 2003). Signal transduction pathways related to theses stresses have been identified in several gene expression studies in plants such as Arabidopsis (Lee and Lee, 2003; Seki et al., 2002) and rice (Rabbani et al., 2003). For sugarcane, a pioneering study has identified several genes induced by low temperature stress (Nogueira et al., 2003). Among these genes, SsNAC23, a homolog of the NAC domain rice transcription factor OsNAC6 was identified. NAC domain proteins constitute a class of plant-specific transcription factors, with important roles in development, growth and response to stress (Kikuchi et al., 2000; Ooka et al., 2003). Members of this family are induced by low temperature stress in rice, by wounding and pathogen attack in potato, and by cold, drought and pathogen in Brassica (Collinge and Bollen, 2001; Hegedus et al., 2003).
The expression of $S s N A C 23$ is strongly induced in sugarcane plants exposed to low temperatures. We have also carried on in situ hybridization studies to identify cell types where expression is most abundant. With the aim of further understanding the regulatory network in response to stress, we used the yeast two-hybrid system to identify sugarcane proteins that interact with SsNAC23.

\section{Materials and methods}

Sugarcane plantlets (Saccharum spp. cv. SP80-3280), propagated axenically in vitro, were maintained as described previously (Nogueira et al., 2005). Plants from the variety Q117 were grown under greenhouse conditions in $200 \mathrm{~mL}$ plastic cups containing a commercial planting mix plus ammonium sulfate and commercial 4-20-20 fertilizer. One month-old plants were used for the cold stress treatments. Plants were either kept at $26^{\circ} \mathrm{C}$ (controls) or transferred to $16^{\circ} \mathrm{C}$ for $24 \mathrm{~h}$ and then to $4^{\circ} \mathrm{C}$ for $48 \mathrm{~h}$, and after that time they were immediately harvested and frozen in liquid $\mathrm{N}_{2}$ and stored at $-80^{\circ} \mathrm{C}$.

For in situ hybridization mature leaf, leaf roll and inflorescence samples were collected from the SP80-380 variety grown in the field, in Piracicaba, state of São Paulo, Brazil (22 $\left.{ }^{\circ} 43^{\prime} \mathrm{S} ; 47^{\circ} 38^{\prime} \mathrm{W}\right)$.

Total RNA was extracted from green parts of sugarcane plants, using the TRIZOL reagent (Invitrogen) with modifications for tissues with a high amount of polysaccharides, according to the manufacturer. The RNA was separated by electrophoresis in a formaldehyde agarose (1\%) gel and transferred to nylon membranes (Hybond N, Amersham Bio- 
sciences, USA). The blots were hybridized with a conserved 3 '-end fragment of $S_{s} N A C 23$, as described previously (Nogueira et al., 2005).

Digoxigenin labeling of RNA probes, tissue preparation, and hybridization conditions were performed as described previously (Gentile et al., 2009). The hybridized tissues were sugarcane mature leaf, leaf roll and mature inflorescence. The hybridized sections were viewed after overnight incubation with the digoxigenin-labeled riboprobe, which contained the Ss $N A C 23$ complete coding sequence. Sections were photographed under anAxiovert 35 microscope (Zeiss, Germany).

A sugarcane cDNA library was constructed using the BDMatchmaker Library Construction and Screening Kit (Clontech, USA). A total of $1 \mu \mathrm{g}$ of mRNA from coldstressed sugarcane plants was used for cDNA synthesis according to the manufacturer's instruction. First strand cDNA was amplified by Long Distance PCR using the BD Advantage 2 PCR Kit (Clontech, USA). The purified library cDNA was then fused to the GAL4 activation domain of pGADT7Rec (Clontech, USA) expressing vector by recombination in the AH109 yeast strain.

The full length coding sequence of SsNAC23 was cloned into pGBKT7 and transformed into the yeast strain Y187. Two hybrid screens were carried out by mating, according to the manufacturer instructions (Clontech, USA). Briefly, an overnight culture of strain Y187 expressing SsNAC23 was mixed with strain AH109, expressing the cDNA library, for $24 \mathrm{~h}$, in rich medium at $30^{\circ} \mathrm{C}$ in a shaker at low rotation (30$50 \mathrm{rpm}$ ). The yeast mixture was plated on synthetic dropout (SD) medium lacking tryptophan, leucine, histidine and adenine to select clones containing proteins that interact with SsNAC23. Positive and negative control analyses were made and plated onto the same SD medium. The mating efficiency and number of screened clones were calculated by plating appropriate dilutions in selective media. Positive colonies appearing within six days of the screen and activating the HIS3 and ADE2 reporter genes were subsequently tested for $\alpha$ galactosidase activity. The interactions were confirmed by restreaking the positive clones onto SD medium lacking tryptophan, leucine, histidine and adenine and containing $\mathrm{x}-\alpha-$ gal to verify the maintenance of the correct phenotype and to test the strength of the interaction.

After the confirmation of the phenotype, plasmid isolation of positive clones was made using a modified lyticase protocol (Philippsen et al., 1991). Because the isolated plasmid DNA was a mixture of both plasmids (bait and library), Escherichia coli cells were transformed by electroporation and plated on LB medium containing ampicillin to select for the $\mathrm{AD} /$ library plasmid only. The cDNA inserts were amplified using the BD Matchmaker AD LD-Insert Screening Amplimer Set (Clontech, USA) and the BD Advantage 2 PCR kit (Clontech, USA). The PCR product was sequenced and then compared with that of other proteins in the databases.

To allow a better understanding of the oxidized and reduced forms of SsNAC23 dimeric structure, a molecular model already described (Nogueira et al., 2005) was submitted for additional energy minimization and equilibrating mo- lecular dynamics simulations. To this end, the Gromacs 4.0.2 program (Van Der Spoel et al., 2005) was used on a quadcore Linux workstation using the GROMOS $53 \mathrm{a} 6$ force field.

Modeled SsNAC23 dimer (as described in Nogueira et al., (2005)) was solvated in a water box using the SPC/E model (Brunne et al., 1993). Reduced and oxidized topologies of the protein were obtained using the pdb2gmx tool. Standard protonation states $(\mathrm{pH}=7.0)$ were used for all residues and protein amino and carboxy-terminals were added. For system neutralization, $\mathrm{Na}^{+}$and $\mathrm{Cl}^{-}$ions were used. Final systems were formed by SsNAC23 dimer in oxidized or reduced forms and 19,480 water molecules. Simulation systems were submitted to a steepest-decent (SD) energy minimization, constraining all protein bonds and water molecules using the LINCS algorithm, and converging to machine precision to remove bad van der Waals contacts. For further relaxation of the system, minimized structure of the complexes was used in unrestrained molecular dynamic for $2 \mathrm{~ns}$ with berendsen-type temperature $(310 \mathrm{~K})$ and pressure (1 atm.) coupling in a NVT ensemble and octahedral simulation cell, implementing the PME method of electrostatic treatment (Essman et al., 1995).

Production dynamics were performed in the same conditions for 15-18 ns. In the trajectory interpretation and analysis, scripts provided with GROMACS 4.0.2 package were used.

\section{Results}

The induction of SsNAC23 expression was observed in plants cultivated in vitro and also in sugarcane plants of a different variety, cultivated in soil (Figure 1). Nogueira et al. (2005) showed that expression of SsNAC23 is induced in

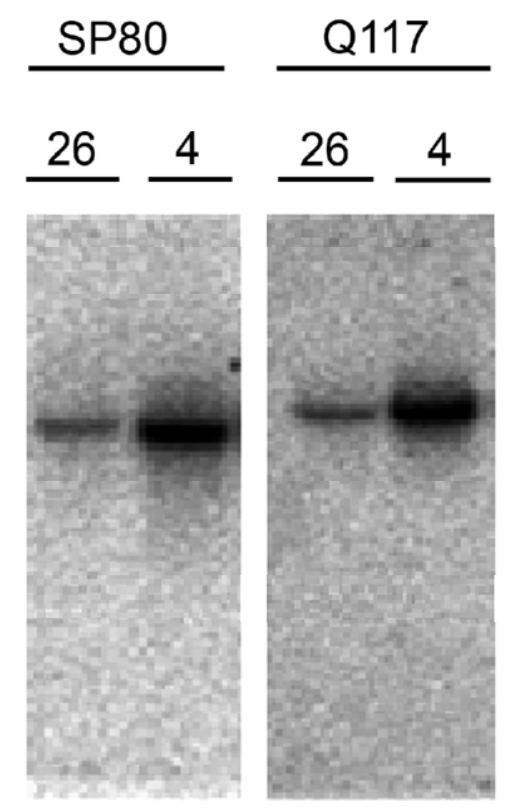

Figure 1- Ss $N A C 23$ expression in response to cold stress. Sugarcane seedlings from in vitro-grown SP80-3280 (SP80) and soil-grown Q117 varieties were cold-stressed at $16^{\circ} \mathrm{C}$ for $24 \mathrm{~h}$ and then $4^{\circ} \mathrm{C}$ for $48 \mathrm{~h}$. Each lane corresponds to RNA extracted from green parts from a single plant. 
sugarcane plantlets cultivated in vitro and exposed to $4^{\circ} \mathrm{C}$ for $48 \mathrm{~h}$. Thus, SsNAC23 cold induction is not an artifact of in vitro cultivation or a specific phenomenon for a particular sugarcane variety, confirming its significance.

Previously, it was demonstrated that SsNAC23 contains a degenerate bipartite nuclear localization signal (NLS), conserved in other NAC protein domain, and that it is localized in the nucleus of onion epidermal cells (Nogueira et al., 2005), further supporting its role as a transcription factor. Regarding tissue expression, $S s N A C 23$ was found to be present in leaves and roots at high to moderate levels, in stems at lower levels, and undetected in the apical meristem and floral organs by northern hybridization (Nogueira et al., 2005). We sought to define even further the localization of $S s N A C 23$ transcripts by in situ hybridization in adult leaf tissue and mature inflorescences from sugarcane. Even though expression in flower organs at a younger stage was undetected by northern hybridization (Nogueira et al., 2005), we decided to further evaluate the expression in this tissue, since the NAC domain proteins are defined by having an important role in meristematic regions and the rice homolog is expressed in flower tissues

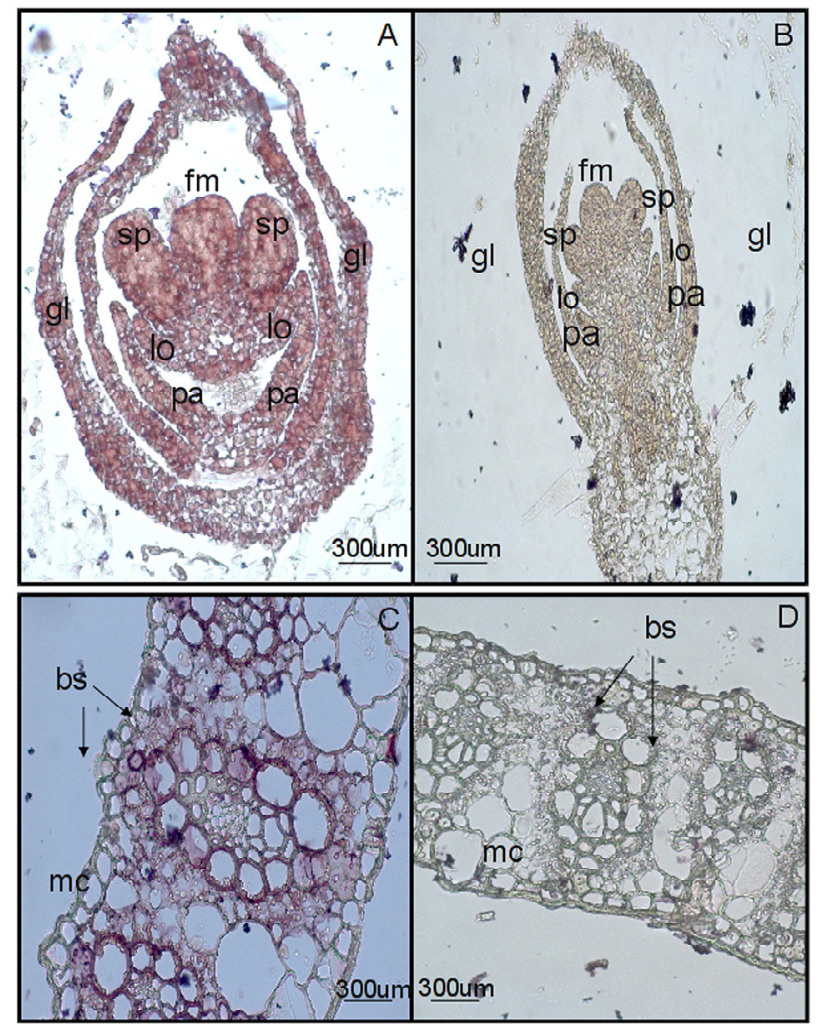

Figure 2 - In situ localization of SsNAC23 transcripts in sugarcane tissues. The hybridization signal with the $S s N A C 23$ probe can be observed as a purple precipitate. Hybridization of longitudinal section of the reproductive meristem with the antisense probe $(\mathrm{A})$ and sense probe (B).gl: glumes; sp: spikelet pair meristem; fm: floral meristem; pa: palea; lo: lodicules. Hybridization of transversal section of the adult leaf with the antisense (C) and sense (D) probe. bs: bundle sheath cells; $\mathrm{x}$ : xylem; p: phloem; mc: mesophyll cells.
(Kikuchi et al., 2000). Indeed, we observed very strong expression of $S_{s} N A C 23$ in the mature sugarcane inflorescence, particularly in the floral meristem (Figures 2A, B). We also observed expression in mature leaves of sugarcane, particularly in metabolic active cells such as the bundle sheath cells (Figures 2C, D).

A better understanding of the role of the SsNAC23 transcription factor during low temperature stress response in sugarcane can be achieved by the identification of additional targets in the cold response pathway. To this end, we performed a yeast two-hybrid assay using SsNAC23 as bait to identify interacting proteins from a cold-stressed cDNA expression library. The full length coding sequence of SsNAC23, expressed as fusion to the binding domain (BD) of the GAL4 protein, did not activate the expression of the reporter genes by itself and it was not toxic to yeast cells (data not shown), both of which could have hindered the yeast two-hybrid approach. The yeast strain expressing SsNAC23 fused to the GAL4 BD was allowed to mate with the strain expressing a cDNA library prepared from RNA from sugarcane plants submitted to $4^{\circ} \mathrm{C}$ for $48 \mathrm{~h}$. After $24 \mathrm{~h}$ of mating, the mixture was plated on selective media and the diploids were allowed to grow for 5 days
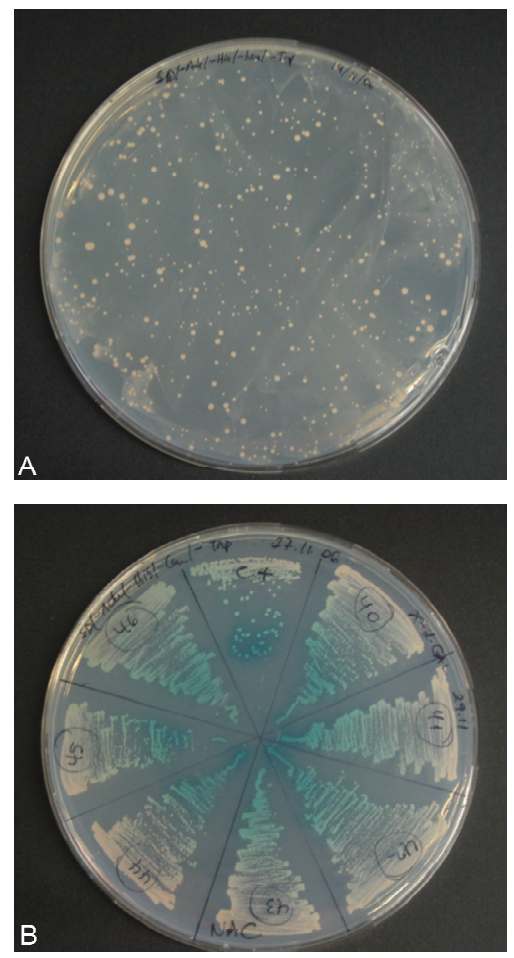

Figure 3 - Yeast two-hybrid screening. Growth of yeast cells in selective media after mating strains Y187 (expressing SsNAC23) and AH109 (expressing sugarcane cDNA library) (A). Growth of yeast cells in selective media containing $\mathrm{X}-\alpha-\mathrm{Gal}$ (positive clones showing $\alpha$ galactosidase activity) (B).The numbers indicate the individual clones that interacted with $S_{s} N A C 23$, grown onto an SD/-Ade/-His/-Leu/-Trp medium. Clones were kept for $5-6$ days at $30^{\circ} \mathrm{C}$ with $300 \mu \mathrm{L} \mathrm{X- \alpha -Gal}$ per plate. 
(Figure 3A). From approximately $7 \times 10^{5}$ colonies screened, about 200 were transferred to selective media containing $x-\alpha-$ gal. These 200 colonies were grown side by side with positive controls (Figure 3B). From these, 150 colonies stained blue and were transferred to selective media with $\mathrm{x}$ - $\alpha$-gal once again, to verify the maintenance of the correct phenotype. A total of 80 colonies maintained the phenotype and 10 of those had their plasmid isolated and sequenced.

A total of ten clones was sequenced and four did not show similarity to any protein in the database. The other six clones, named NAC23ip1 (NAC23 interacting protein number 1), NAC23ip2, NAC23ip3, NAC̈23ip4, NAC23ip5, and NAC23ip6, had similarity with proteins ranging from stressrelated, to nuclear import, to other cellular processes (Table 1). NAC23ip1 encodes a thioredoxin h1 protein homologue involved in protecting cells from reactive oxygen species during stress responses (Meyer et al., 2008). NAC23ip2 codes for a voltage dependent anionic channel (VDAC). Two clones, NAC23ip3 and NAC23ip4, showed similarity to molibdoenzymes belonging to the sulfite oxidase family (SO, responsible for the oxidation of sulfite to sulfate), a recently discovered family in plants (Eillers et al, 2001). NAC23ip5 encodes a homolog of the heat shock protein hsp70 from maize (Rochester et al., 1986), and NAC23ip6 was identified as a ribosomal protein $\mathrm{S} 10 \mathrm{p} / \mathrm{S} 20 \mathrm{e}$.

A thioredoxin h1 was identified as contacting SsNAC23 protein in the yeast two-hybrid system. The sugarcane SAS corresponding to this clone, SCCCLR2001H09.g, has several reads that were sequenced from cDNA libraries from flowers and young leaves, indicating it is co-expressed with Ss $N A C 23$ in several sugarcane tissues, corroborating to the data from protein-protein interactions (data not shown). Thiorredoxins catalyze the reduction/oxidation of disulfide bond dissociations/formation and the possibility of disulfide bridge formation in the SsNAC23 structure was analyzed. For this propose, the SsNAC23 molecular model already obtained by our group (Nogueira et al., 2005) was used as a starting point. Despite the high identity of NAC-like sequences, SsNAC23 protein contains three Cys residues (Cys 29, Cys 32 and Cys 155) but only Cys155 appears to be conserved on the general fold of NAC-like structure (Figure 4). In groups of NAC-like proteins, Cys residues appear at the $\mathrm{N}$-terminal part of the protein in positions between 25 and 60 in a few members of the family (less than $25 \%$ of 500 analyzed sequences). However, unpaired Cys residues in these positions appear to be a characteristic of these groups, as no other Cys residues were observed in other parts of the pro- tein (a clear exception was the highly conserved Cys155 position on this fold) (Figure 4).

We tested the possibility of disulfide bridge formation between Cys29 and Cys32 in SsNAC23 (presented as Cys191 and Cys194 in our dimeric molecular model, Figure 5A). These residues lie in the $\alpha$-helix at the $\mathrm{N}$-terminal part of the structure and are partially exposed to the solvent in the lateral surface of the dimer (Figure 5B). As a consequence, these residues are free for tioredoxin $\mathrm{h} 1$ recognition and redox action. On the top of our dimeric model a large hydrophobic surface was also observed (in green in Figure 5B), which partially co-localized with the proposed Cys29-Cys32 disulfide bridge, suggesting a viable place for protein-protein interactions.

With the aim of further detailed characterization of oxidized (Cys29-Cys32 bridge) and reduced (CysH form) SsNAC23 protein, two different simulation systems were modeled, using water box and molecular dynamic simulations performed for 15 to 20 ns. The trajectories of different experiments were analyzed and the most represented cluster of structures was used in superposition of oxidized and reduced forms of the SsNAC23 structure (Figure 5C). In this superposition, differences are clear in the lateral part and especially on the top of the structure, near the Cys29-Cys32 bridge and covering the central part of the SsNAC23 hydrophobic surface (Figure 5C). Also, residue fluctuation analysis over the simulation trajectory for oxidized and reduced forms of SsNAC23 structures shows pointed differences in residues at 25 to 28 and 33 to 40 positions (Figure 5D).

\section{Discussion}

Members of the NAC family are induced by low temperature, as well as other stresses, in several plant species (Collinge and Bollen, 2001; Hegedus et al., 2003; Nogueira et al., 2005; Pinheiro et al., 2009). Here we observed that the expression of $S s N A C 23$ is induced in sugarcane plants exposed to low temperatures $\left(4^{\circ} \mathrm{C}\right)$. In addition to cold, we showed that $S_{s} N A C 23$ is induced by drought and herbivory in sugarcane (Nogueira et al., 2005).

To further characterize the transcription factor SsNAC23, we have also carried on in situ hybridization studies to identify cell types in tissues where expression is most abundant. The results of these studies showed that the SsNAC23 expression is localized on rapidly dividing cells, such as floral meristem and on metabolic active cells such as bundle sheath cells in mature leaf tissue. Our previous study did not de-

Table 1 -Positive clones identified in the yeast two hybrid analyses using the complete sequence of SsNAC23 as bait.

\begin{tabular}{|c|c|c|}
\hline Clone ID & Best hit ${ }^{\text {(Accession, Score, E-value) }}$ & Description of process involved \\
\hline NAC23ip1 & Thioredoxin h1 (CAI64400, 113, 1e $\left.\mathrm{e}^{-45}\right)$ & Reactive oxygen species formation \\
\hline NAC23ip2 & Voltage-dependent anion channel $\left(469,6 \mathrm{e}^{-131}\right)$ & Mitochondrial membrane protein \\
\hline NAC23ip3 & Putative sulfite oxidase-like protein (NP_001062326, $1302 \mathrm{e}^{-67}$ ) & Peroxisomal protein involved with $\mathrm{SO}_{2}$ detoxification \\
\hline NAC23ip4 & Putative sulfite oxidase-like protein (NP_001062326, 398 2e-109) & Peroxisomal protein involved with $\mathrm{SO}_{2}$ detoxification \\
\hline NAC23ip5 & Heat shock protein HSP70 (CAA27330, 152, 3e-35) & Molecular chaperone involved in nuclear import \\
\hline NAC23ip6 & Ribosomalprotein S10p/S20e (AAV31119, 239, 1e-61) & Ribosome formation \\
\hline
\end{tabular}



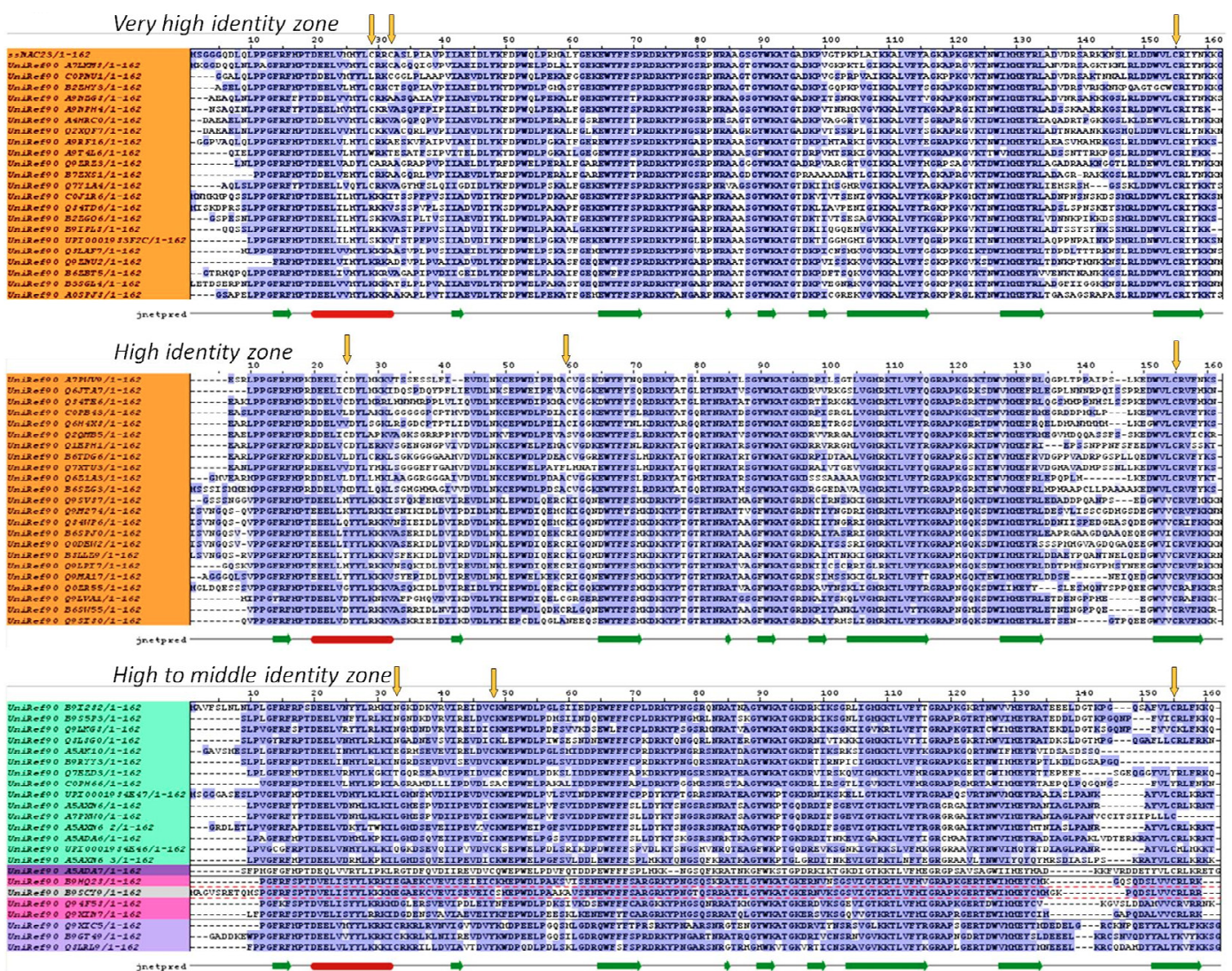

Figure 4 - Parts of sequence alignment of NAC-like proteins with demonstrated identity to our SsNAC23 (AAW62955). Positions presenting some conserved Cys residues were pointed by yellow arrows and sequences were colored in dark (light) blue representing identity (similarity) according to BLOSUM62 matrix. Secondary structure elements were imported from our modeled structure. The total alignment was obtained using JalView 3.0 in a total universe of more than 500 aligned NAC-like proteins (www.jalview.org).

tect $\operatorname{Ss} N A C 23$ expression in flower tissue by northern blot (Nogueira et al., 2005), perhaps because the tissue used in the present study is at an earlier developmental stage than the one used in the previous study, and/or because of the higher sensitivity of the in situ hybridization technique. The stronger expression of $S s N A C 23$ that we observed in the flower meristem is in line with several reports indicating that NAC proteins are involved in flower development (Souer et al., 1996; Aida et al., 1997; 2002).

With the aim of further understanding the regulatory network in response to stress, we used the yeast two-hybrid system to identify sugarcane proteins that interact with SsNAC23. Using SsNAC23 as a bait, we screened a cDNA library of sugarcane plants submitted to $4^{\circ} \mathrm{C}$ for 48 hours. Several interacting partners were identified, including a thioredoxin h1 (clone NAC23ip1). These proteins act in different cellular processes, such as the detoxification of reactive species of oxygen which occur during diverse stress situations (Meyer et al., 2008; Gelhaye et al., 2004). Interestingly, Yan et al. (2006) identified a rice thioredoxin $h$ induced by low temperatures, and Serrato et al. (2003) observed that a thioredoxin $h$ accumulates in the nucleus of wheat seeds under oxidative stress. One possibility for the interaction be- tween the sugarcane thioredoxin and SsNAC23 could be the regulation of disulfide bridges between some of the three cysteine residues in SsNAC23. However, the SsNAC23 homolog from Arabidopsis thaliana, ANAC, does not contain disulfide bridges (Ernst et al., 2004). The differences found in the fluctuation and in the local structure near the Cys29 and Cys32 residues of SsNAC23 indicate a distinct hydrophobic surface and shape (Figure 5D), reflecting in different surface recognition characteristics for oxidized and reduced SsNAC23 forms. All these observations together suggest that the Cys29-Cys31 disulfide bridge as a hot-spot for structural modulation of recognition of SsNAC23 by other proteins. This was observed for other transcription factors, like HLF (Hypoxia-inducible factor 1like), in which a reduction in one cysteine residue in the HLF protein is essential for its interaction with the CBP/ p300 complex (Ema et al., 1999), and this complex regulates several genes (Goodman and Smolik, 2000). The activation of a glicocorticoid receptor (GR) that contains a zinc finger is also modulated by a direct interaction with a thioredoxin, and the oxidative state of the cysteine residues is a potential enzyme target under oxidative stress conditions (Makino et al., 1999). 


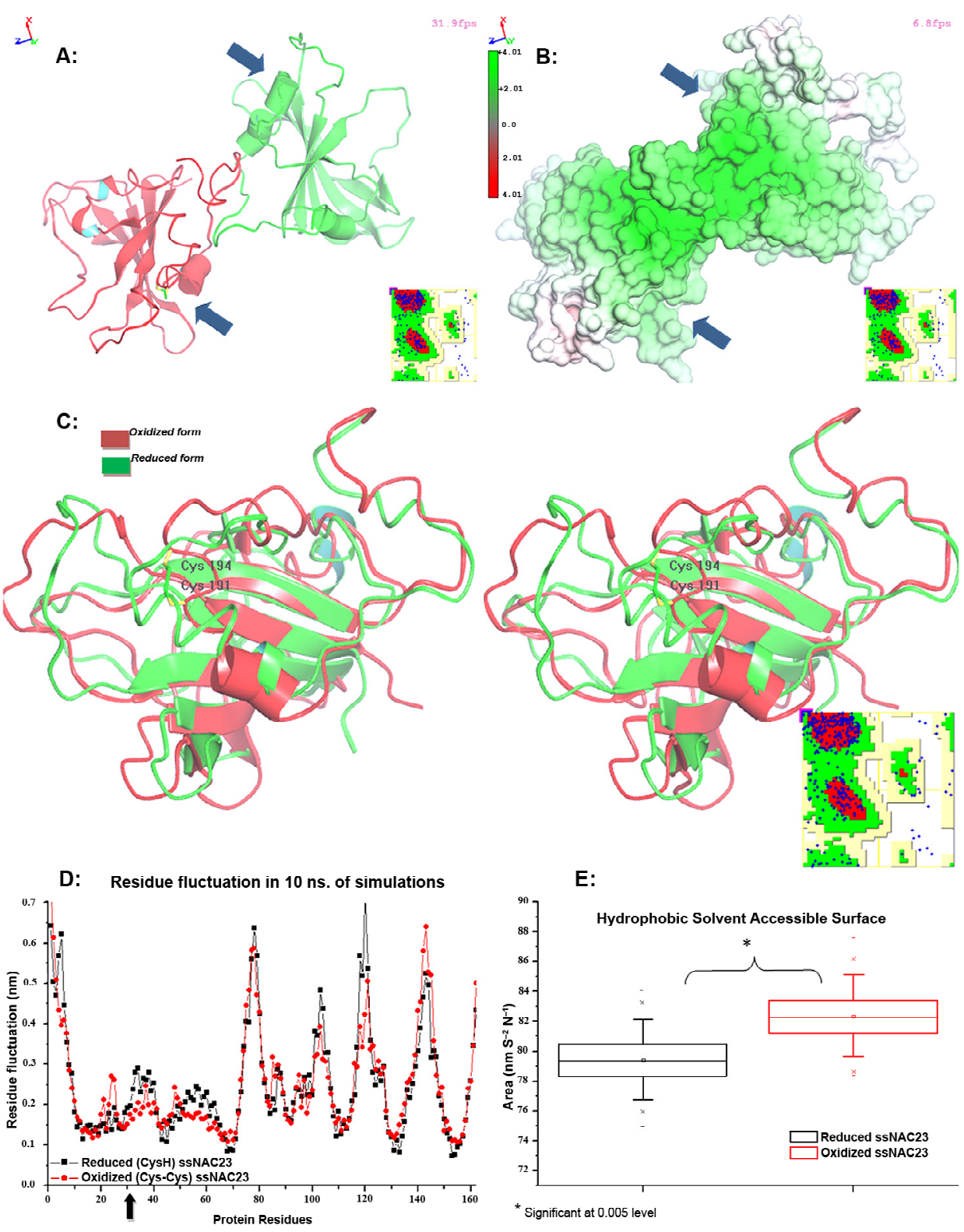

Figure 5 - Reduced and oxidized structures of the $S s N A C 23$ model showed differences in their hydrophobic surfaces. A: Top view of backbone representation of heterodimeric structure of $S s N A C 23$ with (red) and without (green) $\mathrm{Cys}_{29}-\mathrm{Cys}_{32}$ disulfide bridge (blue arrows). B: Same structure as A, but protein surface colored according to hydrophobic characteristics. Big hydrophobic surface (in green) on the top of the $S s N A C 23$ dimeric structure suggest a good place for interaction with other proteins. C: Lateral stereo-representation of structural superposition of reduced (green) and oxidized (red) Ss $N A C 23$ monomers. Cys-Cys bond was represented in wire and numbered according to our modeled structure. D: Calculated residue fluctuation in molecular simulation experiments, using a reduced (black) and oxidized (red) monomers of $S_{s} N A C 23$. The $\mathrm{Cys}_{29}{ }^{-} \mathrm{Cys}_{32}$ disulphide bridge was represented as black arrow. E: Percentage of hydrophobic surface in reduced and oxidized $S$ s $N A C 23$ during 20 ns. simulations.

The clone NAC23ip5 codes for a homolog of the heat shock protein hsp70 from maize (Rochester et al., 1986). Heat shock proteins (HSP) act as chaperones and one of their functions is to facilitate protein transport to the nucleus (Shulga et al., 1996). This hsp70 from sugarcane could be part of the group of proteins responsible for SsNAC23 translocation from the place of synthesis (cytoplasm) to where it functions (nucleus). Curiously, a spinach homologue of the maize hsp70 has increased levels during spinach cold acclimation (Neven et al., 1992). Recently it was demonstrated that this protein, denominated $\mathrm{Hsc} 70$, has chaperone activity in plants of spinach exposed to cold stress (Zhang and Guy, 2006). The authors suggested that during cold stress, Hsc70 could act by avoiding denaturation and protein aggregation at low temperatures. It could be speculated that SsNAC23 is a thermo-sensitive protein that is re-structured by the sugarcane hsp70. 
The clone NAC23ip6 was identified as a ribosomal protein $\mathrm{S} 10 \mathrm{p} / \mathrm{S} 20 \mathrm{e}$. In prokaryotes the protein is named S10, while in eukaryotes it is denominated S20. There are several evidences indicating that the protein $\mathrm{S} 10$ is one of the proteins shared by the transcription and translation machinery (Squires and Zaporojets, 2000). In yeast, the protein S20 is able to suppress a mutation in the RNA polymerase III that cause temperature sensitivity due a direct interaction with the RNA polymerase (Hermann-Le Denmat et al., 1994). Therefore, it is possible that when SsNAC23 interacts with the S20 protein from sugarcane, it could have an effect on the transcription of genes that produces tRNA or rRNA. Recently, Nozawa et al. (2006) showed the role of the protein AtRPS20b from Arabidopsis thaliana in response to stress. These authors overexpressed the protein in yeast and observed a better performance under boric acid stress. In tobacco, Rogalski et al. (2008) demonstrated that Rpl33, a ribosomal protein encoded by the plastid is not essential for cell survival but it is required under cold stress conditions. They suggested that the lack of the ribosomal protein may affect cells under conditions where the maximum translational capacity of the plastid is needed. It is possible that SsNAC23 interacts with these types of proteins under cold stress conditions to maintain a high translational level during reinitiation of protein biosynthesis after cold damage. This subject is relatively new and little is known, and therefore the possibilities are very interesting.

Another clone, NAC23ip2, codes for a voltage dependent anionic channel (VDAC) that is localized in the mitochondria membrane of maize cells (Geiger et al., 1999). These proteins have an important role in the control of metabolites flow between mitochondria and cytoplasm (Colombini, 2004). Curiously, a VDAC from Pennisetum glaucum is induced not only by cold, but also by salinity, drought and by the application of salicylic acid (Desai et al., 2006). However, this protein appears to be localized in the mitochondria and data of subcellular localization obtained previously (Nogueira et al., 2005) showed that $S s N A C 23$ is localized in the nucleus.

Two clones (NAC23ip3 and NAC23ip4) are similar to molibdoenzymes from the sulfite oxidase family (SO). Only recently it was discovered that plants have this enzyme family (Eilers et al., 2001). In Arabidopsis, a SO is localized in peroxisomes and is involved in the oxidation of sulfite to sulfate. The importance of these reactions is based on the toxicity of sulfite, which can break disulfide bridges and inhibit several metabolic reactions. The elimination of sulfite can protect the plant from $\mathrm{SO}_{2}$ present in the atmosphere and produced during decomposition of amino acids containing sulfur (Hansch et al., 2006). However, an association between $\mathrm{SO}_{2}$ and cold stress has not been found, and we suggest that this protein might regulate the SsNAC23 in a way that is unknown so far.

In summary, an important transcription factor associated with stress responses in sugarcane plants was further characterized. We confirmed the previously observed cold-induction of $S s N A C 23$ in plantlets grown in vitro and extended these findings to plants cultivated in soil. We also character- ized the expression of $S s N A C 23$ in cell types of two tissues, the reproductive meristem and adult leaves. To contribute to our understanding of the signal transduction pathway involved in stress, we identified SsNAC23-interacting partners by the yeast two-hybrid assay. One of them, a thioredoxin, might act changing the structure of SsNAC23 with interesting implications for its interactions with other proteins.

\section{Acknowledgements}

This work was supported by grant 04/15865-8 FAPESP to $\mathrm{MM}$ and grant from FAPERJ to JHF. RFD, RGT and SRC received fellowships from FAPESP.

\section{References}

Aida, M.; Ishida, T.; Fukaki, H.; Fujisawa, H.; Tasaka, M. 1997. Genes involved in organ separation in Arabidopsis: an analysis of the cup-shaped cotyledon mutant. Plant Cell 9: 841-857.

Aida, M.; Vernoux, T.; Furutani, M.; Traas, J.; Tasaka, M. 2002.Roles of PIN-FORMED1 and MONOPTEROS in pattern formation of the apical region of the Arabidopsis embryo.Development 129: 3965-3974.

Allen, D.J.; Ort, D.R. 2001.Impacts of chilling temperatures on photosynthesis in warm-climate plants. Trends in Plant Science 6: 36-42.

Brunne, R.M.; Liepinsh, E.; Otting, G.; Wuthrich, K.; Van Gunsteren, W.F. 1993. Hydration of proteins. A comparison of experimental residence times of water molecules solvating the bovine pancreatic trypsin inhibitor with theoretical model calculations. Journal of Molecular Biology 231: 1040-1048.

Collinge, M.; Bollen, T. 2001.Differential induction of two potato genes, Stprx2 and StNAC in response to infection by Phytophthorainfestansand to wounding.Plant Molecular Biology 46: 521-529.

Colombini, M. 2004. VDAC: The channel at the interface between mitochondria and the cytosol. Molecular and Cell Biochemistry256/ 257: 107-115.

Desai, M.K.;Mishra, R.N.; Verma, D.; Nair, S.; Sopory, S.K.; Reddy,M.K. 2006. Structural and functional analysis of a salt stress inducible gene encoding voltage dependent anion channel (VDAC) from pearl millet (Pennisetumglaucum). Plant Physiology and Biochemistry44: 483-493.

Du, Y.C.; Nose, A.; Wasano, K. 1999.Effects of chilling temperature on photosynthetic rates, photosynthetic enzyme activities and metabolite levels in leaves of three sugarcane species. Plant Cell and Environment 22: 317-324.

Eilers, T.; Schwarz, G.; Brinkmann, H.; Witt, C.; Richter, T.; Nieder, J.; Koch, B.; Hille, R.; Hansch, R.; Mendel, R.R. 2001. Identification and biochemical characterization of Arabidopsis thaliana sulfite oxidase: A new player in plant sulfur metabolism. Journal of Biological Chemistry 276: 46989-46994.

Ema, M.; Hirota, K.; Mimura, J.; Abe, H.; Yodoi, J.; Sogawa, K.; Poellinger, L.; Fujii-Kuriyama, Y. 1999. Molecular mechanisms of transcription activation by HLF and HIF1alpha in response to hypoxia: their stabilization and redox signal-induced interaction with CBP/ p300.EMBO Journal 18: 1905-1914.

Ernst, H.A.; Olsen, A.N.; Skriver, K.; Larsen, S.; Lo Leggio, L. 2004. Structure of the conserved domain of ANAC, a member of the NAC family of transcription factors. EMBO Reports 5:297-303.

Essman, U.; Perela, L.; Berkowitz, M.L.; Darden, T.; Lee, H.; Pedersen, L.G. 1995. A smooth particle mesh Ewald method. Journal of Chemical Physics 103: 8577-8592.

Geiger, T.R.; Keith, C.S.; Muszynsky, M.G.; Newton, K.J. 1999.Sequence of three maize cDNAs encoding mitochondrial voltage-dependent anion channels (VDAC) protein.Plant Physiology121: 685-686. 
Gelhaye, E.; Rouhier, N.; Gerard, J.; Jolivet, Y.; Gualbeto, J.; Navrot, N.; Ohlsson, P.I.; Wingsle, G.; Hirasawa, M.; Knaff, D.B.; Wang, H.; Dizengremel, P.; Meyer, Y.; Jacquot, J.P. 2004. A specific form of thioredoxin $\mathrm{h}$ occurs in plant mitochondria and regulates the alternative oxidase. Proceedings of the National Academy of Sciences 101: 14545-14550.

Gentile, A.; Ditt, R.F.; Dias, F.O.; da Silva, M.J.; Dornelas, M.C.; Menossi, M. 2009. Characterization of ScMAT1, a putative TFIIH subunit from sugarcane. Plant Cell Reports 28: 663-672.

Goodman, R.H.; Smolik, S. 2000. CBP/p300 in cell growth, transformation, and development. Genes and Development 14: 1553-1577.

Guy, C.L.; Niemi, K.J.; Brambl, R. 1985.Altered gene expression during cold acclimation of spinach. Proceedings of the National Academy of Sciences 82: 3673-3677.

Hansch, R.; Lang, C.; Riebeseel, E.; Lindigkeit, R.; Gessler, A.; Rennenberg, H.; Mendel, R.R. 2006. Plant Sulfite Oxidase as Novel Producer of $\mathrm{H}_{2} \mathrm{O}_{2}$. Journal of Biological Chemistry 281: 6884-6888.

Hegedus, D.; Yu, M.; Baldwin, D.; Gruber, M.; Sharpe, A.; Parkin, I.; Whitwill, S.; Lydiate, D. 2003. Molecular characterization of Brassica napus NAC domain transcriptional activators induced in response to biotic and abiotic stress. Plant Molecular Biology 53: 383-397.

Hermann-Le Denmat, S.; Werner, M.; Sentenac, A.; Thuriaux, P. 1994. Suppression of yeast RNA polymerase. III Mutations by FHL1, a gene coding for a fork head protein involved in rRNA processing. Molecular and Cell Biology 14:2905-2913.

Kikuchi, K.; Ueguchi-Tanaka, M.; Yoshida, K.T.; Nagato, Y.; Matsusoka, M.; Hirano, H.Y. 2000. Molecular analysis of the NAC gene family in rice.Molecular and General Genetics 262: 1047-1051.

Lee, J.Y.; Lee, D.H. 2003. Use of serial analysis of gene expression technology to reveal changes in gene expression in Arabidopsis pollen undergoing cold stress. Plant Physiology132: 517-529.

Makino, Y.; Yoshikawa, N.; Okamoto, K.; Hirota, K.; Yodoi, J.; Makino, I.; Tanaka, H. 1999. Direct association with Thioredoxin allows Redox regulation of glucocorticoid receptor function. Journal of Biological Chemistry 274: 3182-3188.

Meyer, Y.; Siala, W.; Bashandy, T.; Riondet, C.; Vignols, F.; Reichheld, J.P. 2008. Glutaredoxins and thioredoxins in plants.Biochimica e BiophysicaActa 1783: 589-600.

Neven, L.G.; Haskell, D.W.; Guy, C.L.; Denslow, N.; Klein, P.A.; Green, L.G.; Silverman, A. 1992. Association of 70-kilodalton heat-shock cognate proteins with acclimation to cold.Plant Physiology 99: 1362-1369.

Nogueira, F.T.S.; Schlogl, P.S.; Camargo, S.R.; Fernandez, J.H.; Rosa, V.E.; Pompermayer, P.; Arruda, P. 2005. SsNAC23, a member of the NAC domain protein family, is associated with cold, herbivory and water stress in sugarcane. Plant Science 169: 93-106.

Nogueira, F.T.S.; Rosa, V.E.; Menossi, M.; Ullian, E.C.; Arruda, P. 2003. RNA expression profiles and data mining of sugarcane response to low temperature. Plant Physiology 132: 1811-1824.

Nozawa, A.; Takano, J.; Kobashayi, M.; Von Wiren, N.; Fijuwara, T. 2006. Roles of BOR1, DUR3, and FPS1 in boron transport and tolerance in Saccharomyces cerevisiae.FEMS Microbiology Letters 262:216-222.

Ooka, H.; Satoh, K.; Doi, K.; Nagata, T.; Otomo, Y.; Murakami, K.; Matsubara, K.; Osato, N.; Kawai, J.; Carninci, P.; Hayashizaki, Y.; Suzuki, K.; Kojima, K.; Takahara, Y.; Yamamoto, K.; Kikuchi, S. 2003. Comprehensive Analysis of NAC family genes in Oryza sativa and Arabidopsis thaliana. DNA Research 10: 239-247.

Philippsen, P.; Stotz, A.; Scherf, C. 1991.DNA of Saccharomyces cerevisiae.Methods in Enzymology 164: 169-182.

Pinheiro, G.L.; Marques, C.S.; Costa, M.D.; Reis, P.A.; Alves, M.S.; Carvalho, C.M.; Fietto, L.G.; Fontes, E.P. 2009. Complete inventory of soybean NAC transcription factors: sequence conservation and expression analyses uncover their distinct roles in stress response. Gene 444 1-2: 10-23.
Rabbani, M.A.; Maruyama, K.; Abe, H.; Khan, M.A.; Katsura, K.; Ito, Y.; Yoshiwara, K.; Seki, M.; Shinozaki, K.; Yamaguchi-Shinozaki, K. 2003. Monitoring expression profiles of rice genes under cold, drought, and high-salinity stresses and abscisic acid application using cDNA microarray and RNA gel-blot analyses. Plant Physiology 133: 1755-1767.

Robertson, M.J.; Inman-Bamber, N.G.; Muchow, R.C.; Wood, A.W. 1999.Physiology and productivity of sugarcane with early and mid-season water deficit. Field Crops Research64: 211-227.

Rochester, D.E.; Winer, J.A.; Shah, D.M. 1986.The structure and expression of maize genes encoding the major heat shock protein, hsp70. EMBO Journal 5: 451-458.

Rogalski, M.; Schottler, M.A.; Thiele, W.; Schulze, W.X.; Block, R. 2008. Rp133, a nonessential plastid-encoded ribosomal protein in Tobacco, is required under cold stress conditions. Plant Cell 20: 2221-2237.

Seki, M.; Kamei, A.; Yamaguchi-Shinozaki, K.; Shinozaki, K. 2003. Molecular responses to drought, salinity and frost: common and different paths for plant protection. Current Opinion in Biotechnology 14: 194-199.

Seki, M.; Narusaka, M.; Ishida, J.; Nanjo, T.; Fujita, M.; Oono, Y.; Kamiya, A.; Nakajima, M.; Enju, A.; Sakurai, T.; Satou, M.; Akiyama, K.; Taji, T.; Yamaguchi-Shinozaki, K.; Carninci, P.; Kawai, J.; Hayashizaki, Y.; Shinozaki, K. 2002. Monitoring the expression profiles of 7000 Arabidopsis genes under drought, cold and high-salinity stresses using a full-length cDNA microarray. Plant Journal 31: 279-292.

Serrato, A.J.; Cejudo, F.J. 2003.Type-h thioredoxins accumulate in the nucleus of developing wheat seed tissues suffering oxidative stress.Planta 217: 392-399.

Shinozaki, K.; Yamaguchi-Shinozaki, K.; Seki, M. 2003.Regulatory network of gene expression in the drought and cold stress responses. Current Opinion in Plant Biology 6: 410-417.

Shulga, N.; Roberts, P.; Gu, Z.; Spitz, L.; Tabb, M.M.; Nomura, M.; Goldfarb, D.S. 1996. In vivo nuclear transport kinetics in Saccharomyces cerevisiae: a role for heat shock protein 70 during targeting and translocation. Journal of Cell Biology 135: 329-339.

Silva, M.A.; da Silva, J.A.G.; Enciso, J.; Sharma, V.; Jifon, J. 2008. Yield components as indicators of drought tolerance of sugarcane. Scientia Agricola 65:620-627.

Souer, E.; Van Houwelingen, A.; Kloos, D.; Mol, J.; Koes, R. 1996. The No Apical Meristem gene of Petunia is required for pattern formation in embryos and flowers and is expressed at meristem and primordia boundaries. Cell 85: 159-170.

Squires, C.L.; Zaporojets, D. 2000.Proteins shared by the transcription and translation machines. Annual Review of Microbiology 54: 775-798.

Tai, P.Y.P.; Lentini, R.S. 2002. Freeze Damage of Florida Sugarcane, in Sugarcane Handbook. Gainesville, University of FloridaAgronomy Department, Gainesville, FL, USA.

Tomashow, M.F. 1998. Role of cold-responsive genes in plant freezing tolerance. Plant Physiology118: 1-8.

Van Der Spoel, D.; Lindahl, E.; Hess, B.; Groenhof, G.; Mark, A.E.; Berendsen, H.J. 2005. GROMACS: fast, flexible, and free. Journal of Computational Chemistry 26: 1701-1718.

Yan, S.; Zhang, Q.; Tang, Z.; Su, W.; Sun, W. 2006.Comparative proteomic analysis provides new insights into chilling stress responses in rice. Molecular and Cellular Proteomics 5: 484-496.

Zhang, C.; Guy, C.L. 2006.In vitro evidence of Hsc70 functioning as a molecularchaperone during cold stress. Plant Physiology and Biochemistry 44: 844-850.

Received April 30, 2010

Accepted September 09, 2010 